\title{
Efecto de la depresión y la autoestima en la ideación suicida de adolescentes estudiantes de secundaria y bachillerato en la Ciudad de México
}

\author{
Effects of depression and self-esteem on suicide ideation in high \\ school adolescent students in Mexico City
}

\author{
Marisol Luna-Contreras y Claudio Alberto Dávila-Cervantes
}

Facultad Latinoamericana de Ciencias Sociales, México

\begin{abstract}
Resumen
La conducta suicida se ha incrementado en jóvenes y adolescentes mexicanos. El objetivo fue estudiar el efecto de la autoestima y la depresión en la ideación suicida en adolescentes estudiantes de secundaria y bachillerato de la Ciudad de México (CDMX) en 2012. Se analizó la Encuesta de Consumo de Drogas en Estudiantes en CDMX utilizando análisis de factores confirmatorio y un modelo de ecuaciones estructurales. Las estudiantes tuvieron mayor prevalencia de ideación suicida, depresión y baja autoestima. La autoestima y la depresión tuvieron un efecto directo en la ideación suicida. La autoestima tuvo un efecto negativo sobre la ideación suicida, cuando está mediada por la depresión. Los estudios sobre ideación suicida ayudan a generar evidencia para la planeación, diseño y evaluación de estrategias, políticas y programas de prevención del suicidio.
\end{abstract}

Palabras clave: Ideación, suicidio, México, adolescentes, autoestima, depresión.

Abstract

Suicide behavior has increased in Mexican adolescents and young adults. The objective was to analyze the effect of self-esteem and depression on suicide ideation in Mexico City's junior high and high school teenager students in 2012. We analyzed Mexico City's Student Drug Consumption Survey of 2012 using a confirmatory factor analysis and a structural equation model. Female students had a higher prevalence of suicide ideation, depression and low self-esteem. Self-esteem and depression had a direct effect on suicide ideation. Self-esteem had a negative effect on suicide ideation when it is mediated by depression. Suicide ideation studies help to generate evidence for the planning, design and evaluation of strategies, policies and programs aimed at suicide prevention.

Key words: Ideation, suicide, Mexico, adolescents, self-esteem, depression. 


\section{INTRODUCCIÓN}

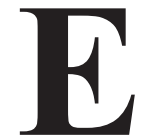

1 suicidio es uno de los principales problemas de salud pública a nivel mundial. Representa cerca de la mitad de las muertes violentas para hombres y más de 70 por ciento para mujeres (OMS, 2014). En 2012 se registraron 80,400 suicidios a nivel mundial, lo que implicó una tasa de 11.4 suicidios por 100 mil habitantes (15 en hombres y ocho por ciento en mujeres) lo que ubicó esta causa de muerte en el decimoquinto lugar (OMS, 2014). Se estima que el suicidio es, además, la segunda causa principal de muerte en la población de 15 a 24 años (adolescentes y adultos jóvenes). Tres de cada cuatro suicidios ocurren en países de ingresos medios y bajos, lo que lleva a estos países a enfrentar una gran carga de mortalidad por esta causa (OMS, 2014).

Por su parte, en México, se ha presentado un incremento de la mortalidad por suicidio desde hace más de 40 años (OMS, 2014; Hernández-Bringas y Flores-Arenales, 2011; Borges et al., 1996; Borges et al., 2010). En 2018 se registraron 6,808 fallecimientos por suicidio, lo que dio por resultado una tasa de mortalidad de 5.43 suicidios por cada cien mil habitantes. Si se compara dicha tasa con aquella observada en 1970 (que ascendió a 1.13 suicidios) (Borges et al., 1996), se puede observar que la tasa de mortalidad casi se cuadruplicó durante este largo periodo. Es importante resaltar que este incremento se presentó principalmente en la población joven (Hernández-Bringas y Flores-Arenales, 2011; Borges et al., 1996; Borges et al., 2010), para quienes el suicidio es la tercera causa de muerte, solo por detrás de los homicidios y accidentes de vehículo automotor; mientras que, entre las edades de diez a diecinueve años, es la primera causa de muerte para mujeres y la tercera para hombres. Más aún, en México el mayor riesgo de conducta suicida se presenta entre los 15 y 19 años de edad (Orozco et al., 2018; Bilsen, 2018).

La conducta suicida se considera un proceso o un continuo que inicia con la ideación, la planificación y el intento; y que culmina con el suicidio consumado (Valdez-Santiago et al., 2018). En este sentido es importante mencionar que la ideación suicida se puede definir como la presencia de pensamientos de falta de valor o deseos de muerte, así como planes o deseos de realizar un suicidio (Harmer et al., 2020). Su estudio es de vital importancia ya que no solo precede al intento de suicidio, sino que es uno de los predictores más importantes del intento (Large et al., 2020; Duarte et al., 2020) y por tanto es uno de los principales factores de riesgo de la mortalidad por suicidio (Kwak e Ickovics, 2019; Chen et al., 2016; OMS, 
2014). La ideación es además un fenómeno común en la población, y que tiene una mayor frecuencia que el intento y que el suicidio consumado, especialmente entre los adolescentes (Rueter et al., 2008). Si no se previene la ideación suicida, ésta puede derivar en un intento de suicidio, tanto así que los adolescentes que han tenido ideación suicida tienen un riesgo 12 veces mayor de suicidio cuando alcanzan los 30 años de edad (Reinherz, Tanner, Berger, Beardslee y Fitzmaurice, 2006).

La adolescencia es un periodo crucial en la vida de las personas, ya que es una etapa de transición hacia la adultez. Esta etapa se caracteriza por ser de una alta vulnerabilidad para el adolescente, durante la cual se incrementa la incidencia de la conducta suicida, es decir, la aparición de los precursores del suicidio consumado como la ideación, la planeación o el intento (Duarte et al., 2020; Bilsen, 2018). De hecho, los adolescentes y jóvenes presentan la mayor incidencia de ideación suicida y tienen un riesgo significativamente mayor de continuar con la planeación y el intento, que en otras etapas de la vida (Crosby, Han, Ortega, Parks y Gfroerer, 2011). El incremento de la conducta suicida en dicha etapa de la vida se ha asociado con el hecho que los adolescentes se encuentran expuestos a una gran cantidad de estresores sociales y dificultades propias de la misma vida, y no son lo suficientemente maduros para enfrentar dichas situaciones (Bilsen, 2018). Por lo que el estudio de la conducta suicida, en particular de la ideación suicida en esta etapa de la vida es un aspecto de gran prioridad (Duarte et al., 2020; Bilsen, 2018).

Los factores que han sido asociados con un mayor riesgo de ideación suicida en la población, y en particular en los adolescentes, son un intento de suicidio previo (OMS, 2014), la presencia de enfermedades mentales o problemas de conducta (Borges et al., 2010), el sexo y la edad(Borges et al., 2017), abuso de sustancias como el alcohol y drogas, tener una baja autoestima, sufrir violencia o abuso, el tipo de funcionamiento familiar, la impulsividad y falta de apoyo social (OMS, 2014). Otros factores menos comunes que también han sido asociados con el suicidio en la adolescencia son la identidad de género (Yadegarfard et al., 2014), la orientación sexual (Baams et al., 2015) y las desventajas socioeconómicas (Yildiz et al., 2018). Entre todos estos factores asociados con el inicio de la conducta suicida en los adolescentes, los que la literatura considera como más importantes son el sexo, la edad, la depresión y la baja autoestima (Lin, 2019).

En cuanto al sexo, si bien el suicidio consumado es más común en hombres, la conducta suicida (que como se mencionó previamente incluye las etapas de ideación, planes e intento) es más frecuente entre las mujeres 
adolescentes mexicanas (Borges et al., 2010). De manera específica, se ha encontrado que las mujeres adolescentes tienen el doble de riesgo que los hombres de la misma edad, de experimentar ideación suicida (Rew, Young, Brown y Rancour, 2016).

La autoestima se ha definido como un sentimiento de auto-aceptación como una persona valiosa e importante, e indica hasta qué punto la persona cree en sus propias capacidades y por lo tanto forma parte de un conjunto de creencias que la persona tiene sobre sí misma (Manani y Sharma, 2016). Esto es, la autoestima es la disposición a considerarse competente frente a los desafíos básicos de la vida y sentirse satisfecho con uno mismo y con sus habilidades (Ceballos-Ospino et al., 2015). Esta definición incluye aspectos cognitivos y afectivos de la autoevaluación, en esencia captura lo que el individuo siente sobre sí mismo (Dinger et al., 2017). La autoestima tiene un impacto significativo en situaciones importantes en la vida, incluyendo la salud y la sociabilización durante la adolescencia y la adultez. De esta manera, existe una conexión entre una alta autoestima y resultados positivos, como éxito laboral, mejores relaciones sociales o una sensación de bienestar (Nguyen et al., 2019). Por el contrario, una baja autoestima se ha relacionado con abuso de sustancia, comportamientos antisociales (Nguyen et al., 2019) y principalmente con la depresión (Rieger et al., 2016; Dinger et al., 2017). A su vez, se ha observado que aquellos adolescentes con mayor autoestima tienden a tener menor conducta suicida, y en particular la baja autoestima tiene una relación fuerte y significativa con la ideación suicida (Primananda y Keliat, 2019). Es por ello que, dicha variable, tiene un rol muy importante en la comprensión del comportamiento suicida en los adolescentes (Manani y Sharma, 2016).

La depresión es un desorden mental que se caracteriza por una tristeza constante y una pérdida de interés en realizar actividades que previamente disfrutaba el individuo, seguida por la incapacidad de llevar a cabo actividades de la vida diaria por al menos dos semanas (WHO, 2016). Esto va unido a sentimiento de poca valía por parte del individuo, lo que se ha estudiado como otro de los principales síntomas de la depresión (Dinger et al., 2019). El riesgo de desarrollar depresión se incrementa en la adolescencia principalmente, y en general presenta una mayor prevalencia en mujeres durante esta etapa de la vida (Breslau et al., 2017). Actualmente, la depresión y sus manifestaciones sintomatológicas son uno de los principales problemas de salud en la población, tanto por su impacto en la vida productiva de los individuos, como porque su prevalencia y la pérdida de años de vida saludables a causa de ella son cada vez mayores (Gonzá- 
lez-Forteza et al., 2011). De igual forma, se ha observado que la depresión es uno de los factores de riesgo más significativos de la ideación suicida en la población (Primananda y Keliat, 2019) y en particular en adolescentes (Hong et al., 2016; Lin, 2019). En este sentido, la depresión incrementa el riesgo de suicidio hasta en 12 veces (Vargas y Saavedra, 2012). Existe evidencia empírica que muestra que la mayoría de la gente que presenta conducta suicida experimenta también depresión (Primananda y Keliat, 2019). Es importante resaltar que la literatura científica indica que tanto la depresión, como la baja autoestima son factores que favorecen la aparición de ideación suicida, especialmente en adolescentes (Ceballos-Ospino et al., 2015). Aunado a ello, se ha observado que existe una correlación negativa entre la autoestima y la depresión, lo que implica que a mayor autoestima tenga una persona, menor será el riesgo de expresar sintomatología depresiva y con ello disminuye el riesgo de desarrollar ideación suicida (Ceballos-Ospino et al., 2015; Nguyen et al., 2019).

La investigación sobre la ideación suicida, y la identificación de sus principales factores de riesgo asociados, resulta de particular importancia en la prevención del suicidio (Bilsen, 2018; Hill et al., 2019). Se ha observado previamente que las personas con pensamientos suicidas suelen tener un mayor riesgo de intento de suicidio, y a su vez, quien lo intenta una vez, es probable que lo intente de nueva cuenta, generalmente con consecuencias fatales (Cha et al., 2018). Es por ello que, para comprender la dinámica relacionada con el crecimiento de la mortalidad por suicidio en México (Borges et al., 2017), es indispensable estudiar la ideación suicida y sus principales factores de riesgo (OMS, 2014; Hill et al., 2019). Además, dichos análisis toman mayor relevancia dado que se ha observado un aumento de la conducta suicida en México, especialmente en la adolescencia (González-Forteza et al., 1998) y particularmente en la zona metropolitana de la Ciudad de México (CDMX) (Borges et al., 2017). Por lo que, el objetivo de la presente investigación, es analizar el efecto de la autoestima y la depresión en la ideación suicida en mujeres y hombres adolescentes estudiantes de secundaria y bachillerato (ESB) de la CDMX en 2012. Con los resultados de este estudio se espera aportar evidencia para tener una mejor comprensión de la ideación suicida en adolescentes en México e identificar áreas de oportunidad para la prevención de dicho fenómeno. Esto es relevante ya que conocer el efecto que tienen dichos factores que influyen en la ideación suicida es útil para tomar acciones apropiadas en la prevención de futuros intentos de suicidios, especialmente en los adolescentes ya que presentan una oportunidad clave para dicha prevención 
que resultará en más años de vida potencialmente preservados (Cha et al., 2018). La importancia de identificar aquellos elementos que incrementan el riesgo de desencadenar un intento de suicidio radica en la posibilidad de implementar acciones necesarias y eficaces para prevenirlo.

\section{Material Y MÉtodos}

\section{Fuente de información y tipo de estudio}

El tipo de investigación que se llevó a cabo es cuantitativo, observacional, de corte transversal, descriptivo y correlacional. La fuente de información utilizada fue la Encuesta de Consumo de Drogas en Estudiantes en Ciudad de México 2012 (ECDE 2012) del Instituto Nacional de Psiquiatría en México. Ésta es una encuesta transversal con representatividad para la CDMX y las 16 demarcaciones territoriales que la conforman, así como de los niveles educación de secundaria y bachillerato.

Para la selección de la muestra, las escuelas se seleccionaron aleatoriamente de cada una de las 16 demarcaciones territoriales. De manera que el diseño de la muestra fue estratificado y por conglomerados. Las características de estratificación fueron el nivel educativo y la demarcación territorial, dando como resultado 32 estratos diferentes, mientras que los conglomerados fueron los grupos escolares (Instituto Nacional de Psiquiatría Ramón de la Fuente, 2013).

La ECDE 2012 utilizó un cuestionario estandarizado y auto-aplicado de manera anónima, el cual ha sido validado en encuestas anteriores (Instituto Nacional de Psiquiatría Ramón de la Fuente Muñiz, 2013). Dada la extensión del cuestionario, los responsables de la ECDE 2012 optaron por dividirlo en siete secciones y cuatro formas que incluía preguntas sobre diferentes temáticas. Estas últimas las nombraron: Forma A, Forma B, Forma C y Forma D (Instituto Nacional de Psiquiatría Ramón de la Fuente Muñiz, 2013).

Es importante mencionar que sólo las siete secciones se aplicaron a todos los estudiantes seleccionados en la muestra, mientras que las cuatro formas sólo se aplicaron a 25 por ciento de los estudiantes de la muestra. Para el caso de este estudio se utilizó la información de las siete secciones comunes a todos los estudiantes y de la forma $\mathrm{A}$. La información recabada en dichas siete secciones es sobre las características sociodemográficas de los estudiantes, sus patrones de consumo de alcohol y drogas, conductas antisociales, nivel satisfacción y nivel de autoestima, por mencionar algunas; mientras que en la Forma A se pregunta sobre la sintomatología de- 
presiva y la ideación suicida, entre otras (Instituto Nacional de Psiquiatría Ramón de la Fuente, 2013).

\section{La ideación suicida y su construcción}

Para determinar la ideación suicida en la población analizada se utilizó la información proveniente de cuatros reactivos de la Forma A, en donde se les preguntó a los estudiantes sobre el número de días (agrupados en 0 , 1-2, 3-4 y 5-7) en los cuales "No podían seguir adelante", "Tenían pensamientos sobre la muerte", "Sentían que mi familia estaría mejor si yo estuviera muerto" y "Pensé en matarme". La agrupación del número de días utilizada, es aquella que el mismo cuestionario tenía originalmente, es decir, no se modificó, sólo se asignó a cada categoría de respuesta un número del 0 al 3, para facilitar a obtención de los resultados (Tabla 1). La elección de las preguntas se hizo con base en las investigaciones realizadas por Roberts (1980), Mariño, Medina-Mora, Chaparro y González-Forteza (1993), González-Forteza y Andrade (1995) y González-Forteza, García, Medina-Mora y Sánchez (1998).

\section{Factores explicativos de la ideación suicida}

Se incorporaron como factores explicativos aquellos que en la revisión de la literatura se consideran como los más relevantes para explicar la ideación suicida entre los jóvenes y adolescentes. En la Tabla 1 se pueden observar tanto la pregunta del cuestionario como las opciones de respuesta. Las opciones de respuesta no se modificaron del cuestionario, pero se les asignó un valor de 0 a 3 en el caso de la ideación suicida, la autoestima y la depresión y de 0 o 1 en la variable de sexo del ESB (Tabla 1). A continuación se mencionan algunas características de los factores explicativos.

- Características sociodemográficas. De los ESB se incluyó el sexo.

- Autoestima. Con base lo propuesto en la escala de autoestima de Rosenberg (1965) y González-Forteza et al. (1997) se seleccionaron cuatro reactivos que permitieran aproximarnos a la autoestima de los ESB.

- Depresión. Para conocer si los ESB presentaban síntomas de depresión se utilizaron seis reactivos propuestos en la escala CES-D (Center for Epidemiologic Studies Depression Scale, por sus siglas en inglés). Estos reactivos permiten observar los síntomas depresivos de los EBS en la semana inmediata anterior a la que se realizó la encuesta. Todo esto 
se hizo con base en lo propuesto por Mariño et al. (1993), Villatoro et al. (2006) y González-Forteza (2011).

Tabla 1: La ideación suicida y sus variables explicativas opciones de respuesta y operacionalización

\begin{tabular}{cc}
\hline Preguntas & Opciones de respuesta \\
en el cuestionario & y operacionalización \\
\hline
\end{tabular}

Ideación suicida

Las siguientes afirmaciones describen forma en que la genta actúa o se siente. Por favor lee cada una y marca el número de días (0 a 7) que

te sentiste así en la última semana.

0: 0 días

1: 1 - 2 días

2: 3 - 4 días

No podía seguir adelante

3: 5 - 7 días

Tenían pensamientos sobre la muerte

Sentían que mi familia estaría mejor si yo estuviera muerto

Pensé en matarme

Sexo

0: Mujer

Autoestima

Por favor indica qué tan acuerdo o en desacuerdo estás con cada una de las siguientes oraciones.

0: Total desacuerdo

Siento que tengo buenas cualidades

1: En desacuerdo

Soy capaz de hacer las cosas tan bien como casi toda la gente

2: De acuerdo

Tengo una actitud positiva hacia mí mismo/a

3: Total de acuerdo

En general estoy satisfecho/a conmigo mis$\mathrm{mo} / \mathrm{a}$

Depresión

Las siguientes afirmaciones describen forma en que la genta actúa o se siente. Por favor lee cada una y marca el número de días (0 a 7) que te sentiste así en la última semana.

0: 0 días

Sentía que no podía quitarme la tristeza ni con la ayuda de mi familia o amigos

1: 1 - 2 días

Me sentí deprimido/a

2: 3 - 4 días

Pensé que mi vida era un fracaso

3: 5 - 7 días

Tenía miedo

Me sentía solo/a

Me sentía triste

Fuente: elaboración propia. 
Efecto de la depresión y la autoestima en la ideación suicida de adolescentes ... / M. LUNA CONTRERAS y C.A. DÁVILA CERVANTES

\section{Población analizada}

La población analizada fueron los ESB de las escuelas públicas y privadas de la CDMX inscritos en el ciclo escolar 2011-2012. El tamaño de la muestra fue de 6,725 estudiantes, con las siguientes características sociodemográficas: 3,365 fueron hombres; 4,632 tenían 15 años o menos, 1,680 entre 16 y 17 años y 413 ESB tenían 18 años o más al momento del levantamiento de la información; y 3,608 cursaban algún año de la secundaria. Este universo de estudiantes corresponde a los que respondieron la Forma A del cuestionario, y que no tuvieron información faltante en las preguntas del cuestionario utilizadas para esta investigación. Es decir, solo se incluyeron los ESB que tuvieron información completa en la estimación del modelo de ecuaciones estructurales, en el análisis factorial y en la parte de estadística descriptiva univariada y bivariada.

\section{Técnicas estadísticas}

Se realizó un análisis descriptivo de las características sociodemográficas utilizando herramientas de estadística descriptiva e inferencial, tales como gráficas de barras, estimación puntual de los porcentajes, los correspondientes intervalos de confianza al 95 por ciento para dichos porcentajes, además de pruebas de hipótesis de diferencias de proporciones para muestras independientes.

Con cada uno de los items de la ideación suicida, la depresión y la autoestima se construyeron variables latentes. Las técnicas estadísticas utilizadas fueron el Análisis de Factores Confirmatorio (AFC) y el Modelo de Ecuaciones Estructurales (SEM, por sus siglas en inglés). Primero, mediante el AFC se construyeron y evaluaron estadísticamente cada uno de los tres constructos (variables latentes) por separado: ideación suicida, autoestima y depresión. Después, con los tres constructos se ajustó el modelo de SEM. Todo se realizó para el total de los ESB y por sexo. Además, se hicieron pruebas de hipótesis estadísticas para corroborar si los coeficientes obtenidos en el modelo de SEM para hombres y mujeres eran estadísticamente diferentes.

Para verificar la confiabilidad de cada constructo latente estimado se utilizó el alfa de Cronbach $(\alpha)$ y el indicador Omega $(\Omega)$. El alfa de Cronbach es una medida de consistencia interna, que puede tomar valores entre 0 y 1. Por su parte, el indicador $W$ propuesto por McDonald (1999) se conoce como una medida de confiabilidad compuesta, que utiliza en su estimación las cargas factoriales del AFC. Se busca que cada constructo 
latente tenga valores mayores o iguales a 0.70 , tanto en el alfa de Cronbach como en el indicador $W$. Esto implica que las variables que lo componen miden el mismo constructo y por tanto la confiabilidad sería alta (Vargas, Delice y Donoso, 2019).

Adicionalmente, para valorar que el ajuste del AFC y del modelo de SEM fuera el óptimo, se utilizaron cuatro medidas de bondad de ajuste: La primera es la Aproximación de la Raíz Cuadrada del Error Cuadrático Medio (RMSEA, por sus siglas en inglés), la cual si tiene un valor inferior 0.05 es indicativo de que el AFC está bien especificado. La segunda media es el Índice de Ajuste Comparativo (CFI, por sus siglas en inglés), mientras que la tercera es el Índice de Tucker-Lewis (TLI, por sus siglas en inglés). Para que los modelos estimados tengan un ajuste óptimo se espera que tanto el CFI y el TLI tengan valores superiores 0.90 (Vargas, Delice y Donoso, 2019). Finalmente, se utilizó la Raíz Cuadrada Media Residual Estandarizada (SRMS, por sus siglas en inglés), donde valores cercanos a 0 son los deseables (López y Zavala, 2019).

Para el procesamiento de la información, recodificación de variables y la obtención de los resultados descriptivos univariados y bivariados se utilizó el paquete estadístico Stata en su versión 15.1. Los resultados del AFC y del modelo de SEM se obtuvieron con el paquete estadístico Mplus8 versión 1.7, mediante el método estimación de máxima verosimilitud y se reportan los coeficientes estandarizados para facilitar la interpretación. Se consideró el factor de expansión de la encuesta para la obtención de todos los resultados.

\section{RESUltados}

La distribución por sexo de la población analizada de ESB fue muy similar, de cada 100 EBS en la CDMX, 51 eran hombres y el resto mujeres. Los estudiantes en su mayoría tenían 17 años o menos y estaban divididos de manera similar entre los niveles de secundaria y bachillerato (Figura 1).

La distribución porcentual de las diferentes variables que integran cada constructo latente analizado para el total y por sexo se muestra en el Tabla 2. Se puede observar que, del total de los ESB, entre 17 y 24 de cada 100 presentó un síntoma de ideación suicida al menos un día de la semana. $\mathrm{Si}$ bien el menos frecuente fue el relacionado con el pensamiento de matarse, 17.1 por ciento de los adolescentes y jóvenes estudiantes de la CDMX mencionaron que pensaron en quitarse la vida, al menos un día a la semana. El síntoma más frecuente que tuvieron los estudiantes, al menos un día a la semana, fue tener pensamientos sobre la muerte (24.1 por ciento). 
Efecto de la depresión y la autoestima en la ideación suicida de adolescentes ... / M. LUNA CONTRERAS y C.A. DÁVILA CERVANTES

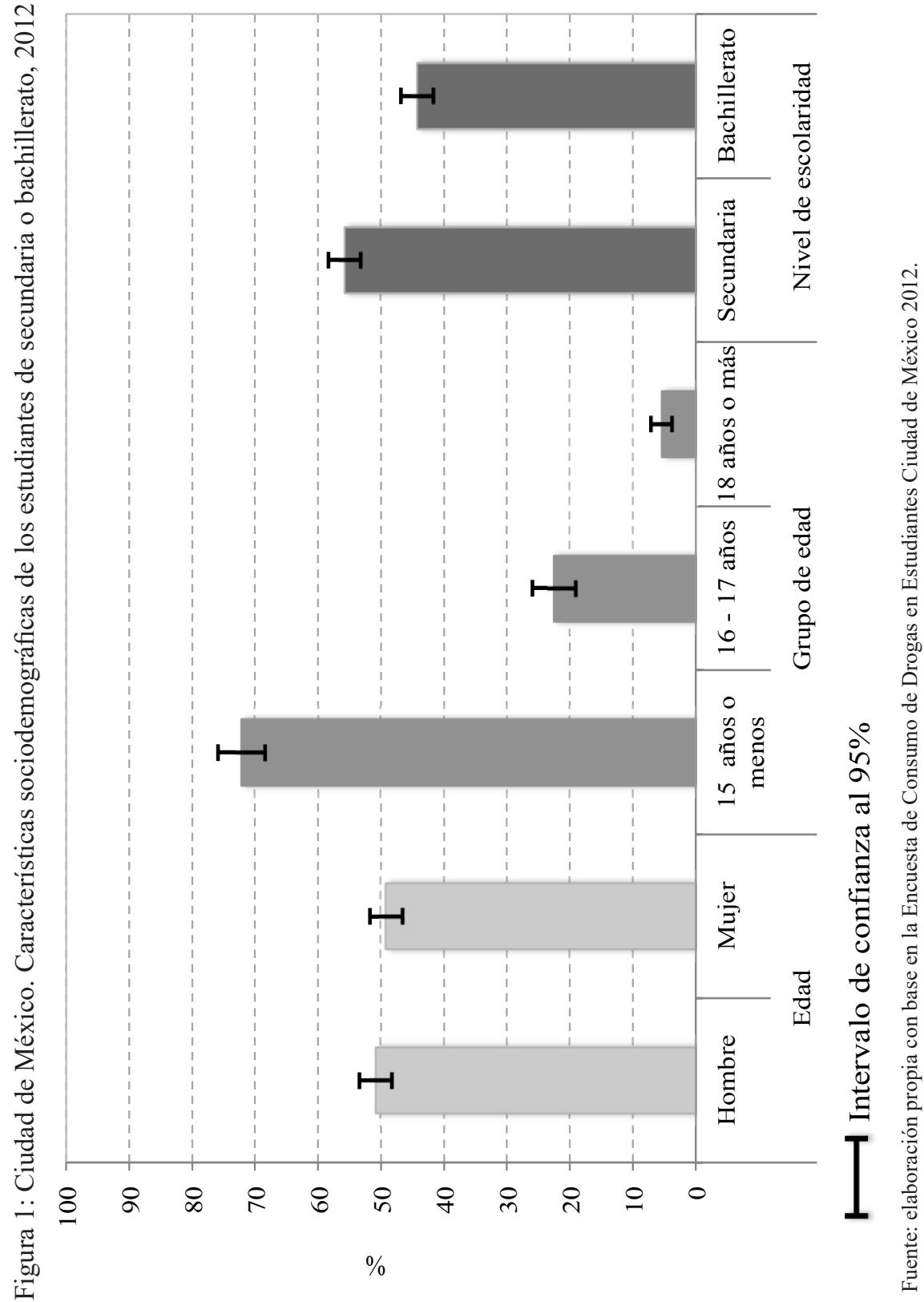


La distribución según el sexo de los ESB es similar, pero destaca que entre las mujeres estudiantes el síntoma de ideación suicida que reportaron con mayor frecuencia fue que "No podía seguir adelante" (con 26 por ciento) por lo menos un día a la semana; mientras que entre sus pares del sexo masculino fue "Tenían pensamientos sobre la muerte" con 23.9 por ciento (Tabla 2).

Tabla 2: Ciudad de México. Distribución porcentual de los componentes de la ideación suicida, depresión y autoestima de los estudiantes de secundaria y bachillerato para el total y por sexo, 2012

\begin{tabular}{|c|c|c|c|c|c|}
\hline \multirow[t]{2}{*}{ Dimensión } & \multicolumn{5}{|c|}{ Total } \\
\hline & 0 días & 1 - 2 días & 3 - 4 días & 5 - 7 días & Total \\
\hline \multicolumn{6}{|l|}{ Ideación suicida } \\
\hline No podía seguir adelante & 76.2 & 12.8 & 5.5 & 5.5 & 100.0 \\
\hline $\begin{array}{l}\text { Tenían pensamientos sobre } \\
\text { la muerte }\end{array}$ & 75.9 & 12.0 & 5.5 & 6.6 & 100.0 \\
\hline $\begin{array}{l}\text { Sentían que mi familia } \\
\text { estaría mejor si yo estuviera } \\
\text { muerto }\end{array}$ & 77.3 & 10.9 & 5.3 & 6.4 & 100.0 \\
\hline Pensé en matarme & 82.9 & 8.5 & 3.5 & 5.2 & 100.0 \\
\hline \multicolumn{6}{|l|}{ Autoestima } \\
\hline $\begin{array}{l}\text { Siento que tengo buenas } \\
\text { cualidades }\end{array}$ & 9.9 & 7.2 & 44.4 & 38.5 & 100.0 \\
\hline $\begin{array}{l}\text { Soy capaz de hacer las cosas } \\
\text { tan bien como casi toda la } \\
\text { gente }\end{array}$ & 8.5 & 8.0 & 36.2 & 47.3 & 100.0 \\
\hline $\begin{array}{l}\text { Tengo una actitud positiva } \\
\text { hacia mí mismo/a }\end{array}$ & 10.1 & 9.0 & 35.4 & 45.5 & 100.0 \\
\hline $\begin{array}{l}\text { En general estoy } \\
\text { satisfecho/a conmigo } \\
\text { mismo/a }\end{array}$ & 10.0 & 8.4 & 33.3 & 48.3 & 100.0 \\
\hline \multicolumn{6}{|l|}{ Depresión } \\
\hline $\begin{array}{l}\text { Sentía que no podía quitar- } \\
\text { me la tristeza ni con la ayu- } \\
\text { da de mi familia o amigos }\end{array}$ & 65.7 & 18.5 & 8.3 & 7.5 & 100.0 \\
\hline Me sentí deprimido/a & 57.2 & 23.3 & 10.8 & 8.7 & 100.0 \\
\hline $\begin{array}{l}\text { Pensé que mi vida era un } \\
\text { fracaso }\end{array}$ & 72.2 & 15.0 & 6.7 & 6.1 & 100.0 \\
\hline Tenía miedo & 66.2 & 20.7 & 7.2 & 5.9 & 100.0 \\
\hline Me sentía solo/a & 62.8 & 20.3 & 8.8 & 8.1 & 100.0 \\
\hline Lloraba a ratos & 66.3 & 17.2 & 7.8 & 8.7 & 100.0 \\
\hline Me sentía triste & 60.0 & 21.8 & 9.4 & 8.8 & 100.0 \\
\hline
\end{tabular}

Fuente: elaboración propia con base en la Encuesta de Consumo de Drogas en Estudiantes Ciudad de México 2012. 
Efecto de la depresión y la autoestima en la ideación suicida de adolescentes ... / M. LUNA CONTRERAS y C.A. DÁVILA CERVANTES

Tabla 2: Continuación

\begin{tabular}{|c|c|c|c|c|c|}
\hline \multirow[t]{2}{*}{ Dimensión } & \multicolumn{5}{|c|}{ Hombres } \\
\hline & 0 días & 1 - 2 días & 3 - 4 días & $5-7$ días & Total \\
\hline \multicolumn{6}{|l|}{ Ideación suicida } \\
\hline No podía seguir adelante & 78.4 & 11.8 & 5.3 & 4.5 & 100.0 \\
\hline $\begin{array}{l}\text { Tenían pensamientos sobre } \\
\text { la muerte }\end{array}$ & 76.1 & 12.2 & 5.6 & 6.1 & 100.0 \\
\hline $\begin{array}{l}\text { Sentían que mi familia } \\
\text { estaría mejor si yo } \\
\text { estuviera muerto }\end{array}$ & 80.2 & 9.8 & 5.1 & 4.9 & 100.0 \\
\hline Pensé en matarme & 84.2 & 7.8 & 3.7 & 4.3 & 100.0 \\
\hline \multicolumn{6}{|l|}{ Autoestima } \\
\hline $\begin{array}{l}\text { Siento que tengo buenas } \\
\text { cualidades }\end{array}$ & 11.0 & 6.0 & 43.1 & 40.0 & 100.0 \\
\hline $\begin{array}{l}\text { Soy capaz de hacer las } \\
\text { cosas tan bien como casi } \\
\text { toda la gente }\end{array}$ & 9.2 & 7.1 & 35.8 & 47.8 & 100.0 \\
\hline $\begin{array}{l}\text { Tengo una actitud positiva } \\
\text { hacia mí mismo/a }\end{array}$ & 10.8 & 6.9 & 34.4 & 47.9 & 100.0 \\
\hline $\begin{array}{l}\text { En general estoy satisfe- } \\
\text { cho/a conmigo mismo/a }\end{array}$ & 10.3 & 6.6 & 31.3 & 51.8 & 100.0 \\
\hline \multicolumn{6}{|l|}{ Depresión } \\
\hline $\begin{array}{l}\text { Sentía que no podía } \\
\text { quitarme la tristeza ni con } \\
\text { la ayuda de mi familia o } \\
\text { amigos }\end{array}$ & 71.1 & 16.4 & 6.9 & 5.6 & 100.0 \\
\hline Me sentí deprimido/a & 63.6 & 21.9 & 8.5 & 6.0 & 100.0 \\
\hline $\begin{array}{l}\text { Pensé que mi vida era un } \\
\text { fracaso }\end{array}$ & 74.7 & 14.0 & 6.4 & 4.8 & 100.0 \\
\hline Tenía miedo & 71.4 & 18.3 & 5.6 & 4.7 & 100.0 \\
\hline Me sentía solo/a & 67.6 & 19.0 & 7.5 & 5.9 & 100.0 \\
\hline Lloraba a ratos & 76.0 & 13.1 & 5.3 & 5.6 & 100.0 \\
\hline Me sentía triste & 68.4 & 18.1 & 7.3 & 6.1 & 100.0 \\
\hline
\end{tabular}

Fuente: elaboración propia con base en la Encuesta de Consumo de Drogas en Estudiantes Ciudad de México 2012. 
Tabla 2: Continuación

\begin{tabular}{|c|c|c|c|c|c|}
\hline \multirow{2}{*}{$\begin{array}{l}\text { Dimensión } \\
\text { Ideación suicida }\end{array}$} & \multicolumn{5}{|c|}{ Mujeres } \\
\hline & 0 días & 1 - 2 días & 3 - 4 días & 5 - 7 días & Total \\
\hline No podía seguir adelante & 74.0 & 13.9 & 5.7 & 6.5 & 100.0 \\
\hline $\begin{array}{l}\text { Tenían pensamientos sobre } \\
\text { la muerte }\end{array}$ & 75.6 & 11.8 & 5.5 & 7.2 & 100.0 \\
\hline $\begin{array}{l}\text { Sentían que mi familia } \\
\text { estaría mejor si yo } \\
\text { estuviera muerto }\end{array}$ & 74.3 & 12.1 & 5.6 & 8.0 & 100.0 \\
\hline Pensé en matarme & 81.5 & 9.1 & 3.3 & 6.0 & 100.0 \\
\hline \multicolumn{6}{|l|}{ Autoestima } \\
\hline $\begin{array}{l}\text { Siento que tengo buenas } \\
\text { cualidades }\end{array}$ & 8.9 & 8.4 & 45.8 & 37.0 & 100.0 \\
\hline $\begin{array}{l}\text { Soy capaz de hacer las } \\
\text { cosas tan bien como casi } \\
\text { toda la gente }\end{array}$ & 7.8 & 8.9 & 36.5 & 46.8 & 100.0 \\
\hline $\begin{array}{l}\text { Tengo una actitud } \\
\text { positiva hacia mí mismo/a }\end{array}$ & 9.4 & 11.2 & 36.4 & 43.0 & 100.0 \\
\hline $\begin{array}{l}\text { En general estoy satisfe- } \\
\text { cho/a conmigo mismo/a }\end{array}$ & 9.7 & 10.3 & 35.4 & 44.7 & 100.0 \\
\hline \multicolumn{6}{|l|}{ Depresión } \\
\hline $\begin{array}{l}\text { Sentía que no podía qui- } \\
\text { tarme la tristeza ni con la } \\
\text { ayuda de mi familia } \\
\text { o amigos }\end{array}$ & 60.2 & 20.5 & 9.7 & 9.5 & 100.0 \\
\hline Me sentí deprimido/a & 50.6 & 24.7 & 13.2 & 11.4 & 100.0 \\
\hline $\begin{array}{l}\text { Pensé que mi vida era } \\
\text { un fracaso }\end{array}$ & 69.6 & 15.9 & 7.0 & 7.5 & 100.0 \\
\hline Tenía miedo & 60.9 & 23.1 & 8.9 & 7.2 & 100.0 \\
\hline Me sentía solo/a & 57.8 & 21.8 & 10.1 & 10.3 & 100.0 \\
\hline Lloraba a ratos & 56.3 & 21.5 & 10.4 & 11.8 & 100.0 \\
\hline Me sentía triste & 51.2 & 25.5 & 11.6 & 11.6 & 100.0 \\
\hline
\end{tabular}

Con respecto a las variables correspondientes al constructo latente de la autoestima, en la Tabla 2 se observa que entre 8.5 y 10.1 por ciento de los ESB reportaron estar en total desacuerdo con las afirmaciones relacionadas con la autoestima, donde "Tengo una actitud positiva hacia mí mismo/a" y 
"En general estoy satisfecho/a conmigo mismo/a" fueron las afirmaciones con mayor porcentaje en esta categoría. Los resultados muestran que los hombres respondieron con mayor frecuencia estar en total desacuerdo con las cuatros variables relacionadas con la autoestima, en comparación con sus pares mujeres (Tabla 2).

Respecto a la sintomatología depresiva se observó que por lo menos 28 de cada 100 ESB de la CDMX reportaron tener estos síntomas una vez a la semana o más. Las estudiantes fueron las que presentan los mayores porcentajes de síntomas relacionados con la depresión al menos un día semana, esto al compararlo con el grupo de estudiantes hombres. El síntoma más frecuente entre las mujeres y los hombres estudiantes fue sentirse deprimido. Llama la atención que, en las mujeres, la mayoría de los síntomas superan 40 por ciento, sobresaliendo "me sentía triste", "lloraba a ratos" y "me sentía sola" (Tabla 2).

En la Figura 2 se observa que 35 de cada 100 ESB presentaron por lo menos un síntoma de ideación suicida al menos una vez a la semana. Lo anterior fue más frecuente entre las mujeres ESB que entre sus pares masculinos, con una diferencia de 4.7 puntos porcentuales. La presencia de uno o más síntomas relacionados con la baja autoestima en uno o más días a la semana fue reportada por 28 por ciento de los ESB; siendo ésta mayor en las mujeres. Del total de ESB poco más de 60 por ciento dijo haber tenido por lo menos un síntoma depresivo un día o más a la semana. La sintomatología depresiva se observó más frecuentemente entre las mujeres que entre los hombres. Destaca que las diferencias entre los porcentajes observados de la ideación suicida, baja autoestima y sintomatología depresiva entre las y los ESB de la CDMX son estadísticamente significativas a 95 por ciento de confianza (Figura 2).

Los resultados de los AFC para los tres constructos latentes (ideación suicida, depresión y autoestima) muestran que tuvieron una buena consistencia interna tanto para el total de los ESB como por sexo, ya que tanto el alfa de Cronbach, como el indicador $\Omega$ tienen valores superiores a 0.80 . Además, los cuatros parámetros de bondad de ajuste de los tres constructos latentes se encuentran en los límites aceptables (Tabla 3). En el constructo latente de ideación suicida, tanto para el total de los ESB como por sexo, la variable observada que más contribuyó fue "Sentían que mi familia estaría mejor si yo estuviera muerto". Por su parte, en la variable latente de autoestima, en todos los casos, la característica con una mayor contribución estadística fue "Tengo una actitud positiva hacia mí mismo/a". 

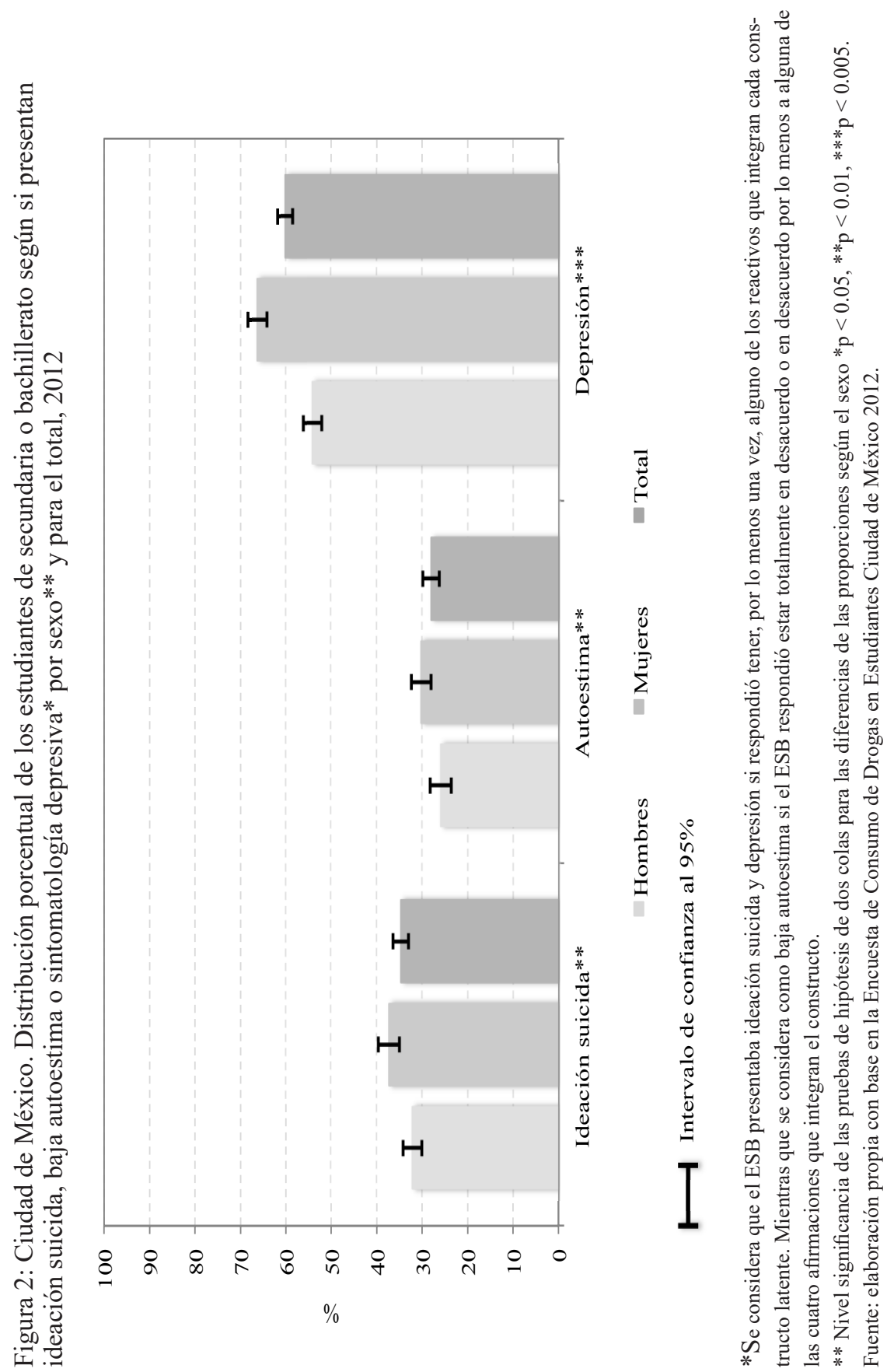
Al considerar a todos los ESB y en hombres el síntoma depresivo con mayor carga factorial en la percepción de la depresión fue "Me sentí deprimido/a", mientras que para el grupo de las mujeres estudiantes fue "Me sentía triste" (Tabla 3).

Tabla 3: Ciudad de México. Coeficientes estandarizados del Análisis Factorial Confirmatorio de cada variable latente para estudiantes de secundaria o bachillerato por sexo y para el total, 2012

\begin{tabular}{|c|c|c|c|}
\hline \multirow{2}{*}{ Variable latente } & \multicolumn{3}{|c|}{$\beta$ estandarizado } \\
\hline & Total & Hombres & Mujeres \\
\hline \multicolumn{4}{|l|}{ Ideación suicida } \\
\hline No podía seguir adelante & $\begin{array}{c}0.738 * * * * \\
(0.015)\end{array}$ & $\begin{array}{c}0.748 * * * * \\
(0.019)\end{array}$ & $\begin{array}{c}0.733 * * * * \\
(0.017)\end{array}$ \\
\hline Tenían pensamientos sobre la muerte & $\begin{array}{l}0.829 * * * * \\
(0.012)\end{array}$ & $\begin{array}{l}0.805 * * * * \\
(0.017)\end{array}$ & $\begin{array}{l}0.848 * * * * \\
(0.014)\end{array}$ \\
\hline $\begin{array}{l}\text { Sentían que mi familia estaría mejor si yo } \\
\text { estuviera muerto }\end{array}$ & $\begin{array}{l}0.862 * * * * \\
(0.011)\end{array}$ & $\begin{array}{l}0.861 * * * * \\
(0.015)\end{array}$ & $\begin{array}{l}0.862 * * * * \\
(0.014)\end{array}$ \\
\hline Pensé en matarme & $\begin{array}{c}0.831 * * * * \\
(0.011)\end{array}$ & $\begin{array}{c}0.829 * * * * \\
(0.015)\end{array}$ & $\begin{array}{c}0.832 * * * * \\
(0.013)\end{array}$ \\
\hline \multicolumn{4}{|c|}{$\begin{array}{l}\text { Total: } \alpha \text { de Cronbach }=0.883 ; \Omega=0.888 ; \text { RMSEA }=0.039, \text { CFI }=0.991 ; \text { TLI }=0.974 ; \\
\text { SRMR }=0.014 . \\
\text { Por sexo: } \alpha \text { de Cronbach de los hombres }=0.880 ; \alpha \text { de Cronbach de las mujeres }=0.886 ; \Omega \text { hombres } \\
=0.885 ; \Omega \text { mujeres }=0.887 ; \text { RMSEA }=0.035, \mathrm{CFI}=0.982 ; \mathrm{TLI}=0.978 ; \text { SRMR }=0.023 .\end{array}$} \\
\hline \multicolumn{4}{|l|}{ Autoestima } \\
\hline Siento que tengo buenas cualidades & $\begin{array}{l}0.812 * * * * \\
(0.010)\end{array}$ & $\begin{array}{l}0.828 * * * * \\
(0.013)\end{array}$ & $\begin{array}{l}0.796 * * * * \\
(0.012)\end{array}$ \\
\hline $\begin{array}{l}\text { Soy capaz de hacer las cosas tan bien } \\
\text { como casi toda la gente }\end{array}$ & $\begin{array}{l}0.832 * * * * \\
(0.009)\end{array}$ & $\begin{array}{l}0.859 * * * * \\
(0.011)\end{array}$ & $\begin{array}{l}0.806 * * * * \\
(0.011)\end{array}$ \\
\hline $\begin{array}{l}\text { Tengo una actitud positiva hacia mí } \\
\text { mismo/a }\end{array}$ & $\begin{array}{l}0.898 * * * * \\
(0.007)\end{array}$ & $\begin{array}{l}0.909 * * * * \\
(0.008)\end{array}$ & $\begin{array}{l}0.886 * * * * \\
(0.010)\end{array}$ \\
\hline $\begin{array}{l}\text { En general estoy satisfecho/a conmigo } \\
\text { mismo/a }\end{array}$ & $\begin{array}{l}0.872 * * * * \\
(0.008)\end{array}$ & $\begin{array}{l}0.876^{* * * * *} \\
(0.010)\end{array}$ & $\begin{array}{l}0.866 * * * * \\
(0.010)\end{array}$ \\
\hline \multicolumn{4}{|c|}{$\begin{array}{l}\text { Total: } \alpha \text { de Cronbach }=; \Omega=0.915 ; \text { RMSEA }=0.097, \mathrm{CFI}=0.974 ; \mathrm{TLI}=0.921 ; \mathrm{SRMR}=0.022 . \\
\text { Por sexo: } \alpha \text { de Cronbach de los hombres }=0.925 ; \alpha \text { de Cronbach de las mujeres }=0.940 ; \Omega \text { hombres } \\
=0.925 ; \Omega \text { mujeres }=0.905 ; \mathrm{RMSEA}=0.079, \mathrm{CFI}=0.992 ; \mathrm{TLI}=0.998 ; \mathrm{SRMR}=0.020 .\end{array}$} \\
\hline \multicolumn{4}{|l|}{ Depresión } \\
\hline $\begin{array}{l}\text { Sentía que no podía quitarme la tristeza } \\
\text { ni con la ayuda de mi familia o amigos }\end{array}$ & $\begin{array}{l}0.764 * * * * \\
(0.011)\end{array}$ & $\begin{array}{l}0.734 * * * * \\
(0.016)\end{array}$ & $\begin{array}{l}0.780 * * * * \\
(0.012)\end{array}$ \\
\hline Me sentí deprimido/a & $\begin{array}{l}0.816^{* * * * *} \\
(0.010)\end{array}$ & $\begin{array}{l}0.797 * * * * \\
(0.013)\end{array}$ & $\begin{array}{l}0.825 * * * * \\
(0.011)\end{array}$ \\
\hline Pensé que mi vida era un fracaso & $\begin{array}{c}0.692 * * * * \\
(0.013)\end{array}$ & $\begin{array}{l}0.682 * * * * \\
(0.018)\end{array}$ & $\begin{array}{c}0.693 * * * * \\
(0.014)\end{array}$ \\
\hline Tenía miedo & $\begin{array}{c}0.682 * * * * \\
(0.014)\end{array}$ & $\begin{array}{c}0.673 * * * * \\
(0.019)\end{array}$ & $\begin{array}{c}0.683 * * * * \\
(0.014)\end{array}$ \\
\hline Me sentía solo/a & $\begin{array}{c}0.765^{* * * * *} \\
(0.011)\end{array}$ & $\begin{array}{c}0.738 * * * * * \\
(0.016)\end{array}$ & $\begin{array}{c}0.778 * * * * \\
(0.012)\end{array}$ \\
\hline Me sentía triste & $\begin{array}{c}0.814 * * * * * \\
(0.009)\end{array}$ & $\begin{array}{c}0.787 * * * * \\
(0.014)\end{array}$ & $\begin{array}{c}0.831 * * * * \\
(0.011)\end{array}$ \\
\hline
\end{tabular}

Total: $\alpha$ de Cronbach $=0.92 ; \Omega=0.889 ;$ RMSEA $=0.048 ; \mathrm{CFI}=0.975 ; \mathrm{TLI}=0.958 ; \mathrm{SRMR}=0.026$. Por sexo: $\alpha$ de Cronbach de los hombres $=0.874 ; \alpha$ de Cronbach de las mujeres $=0.894 ; \Omega$ hombres $=0.876 ; \Omega$ mujeres $=0.895 ;$ RMSEA $=0.045$, CFI $=0.965 ; \mathrm{TLI}=0.962 ; \mathrm{SRMR}=0.034$.

$*$ p-valor $\leq 0.10 ; * *$ p-valor $\leq 0.05 ; * * *$ p-valor $\leq 0.01 ; * * *$ p-valor $\leq 0.001$.

Error estándar entre paréntesis.

Fuente: elaboración propia con base en la Encuesta de Consumo de Drogas en Estudiantes Ciudad de México 2012. 
Los modelos de SEM ajustados de la ideación suicida y la autoestima, mediados por la depresión, tuvieron un buen ajuste global, tanto para el modelo que incluye a todos de los ESB, como el realizado por sexo. Los resultados muestran que, entre los ESB de la CDMX, la autoestima y la depresión tienen un efecto directo y estadísticamente significativo en la ideación suicida, pero este efecto es diferente en cuanto a magnitud y sentido (Figura 1). De igual forma, la depresión tuvo una relación positiva con la ideación suicida, de manera que al aumentar los días que los estudiantes presentan síntomas depresivos aumentará en 0.752 unidades estándar la ideación suicida. La autoestima por su lado tuvo un efecto negativo en la ideación suicida, lo que significa que en la medida que los estudiantes presentan una mayor autoestima, disminuye la ideación suicida en 0.067 unidades estándar. De esta forma, la depresión resultó ser la característica que tiene un mayor efecto sobre la ideación suicida, ya que su coeficiente estandarizado es 12.5 veces mayor que el coeficiente de autoestima. Adicionalmente, se observa que la autoestima tuvo un efecto negativo en la depresión de los ESB. Dicho efecto indica que entre menor sea la autoestima mayor será la sintomatología depresiva; dicho efecto resultó ser de 0.222 unidades estándar.

El sentido de las relaciones encontradas para el conjunto de ESB de la CDMX se preservan en los resultados encontrados por sexo, pero no la magnitud de éstas, ni la significancia estadística. Por un lado, entre los estudiantes hombres, la depresión tuvo un mayor efecto sobre la ideación suicida que entre sus pares mujeres, diferencia que es estadísticamente significativa. Dicho efecto en los hombres ESB fue que por cada unidad que aumente la depresión, la ideación suicida se incrementó en 0.817 unidades estándar; mientras que entre las mujeres la ideación suicida aumentó 0.696 unidades estándar. Por otro lado, aun cuando el efecto de la autoestima sobre la ideación suicida entre las mujeres ESB fue relativamente pequeño, éste sí resultó ser estadísticamente significativo. Sin embargo, no fue así entre el grupo de los estudiantes hombres (Tabla 4).

Por otro lado, la autoestima tanto entre las y los ESB mostró tener un efecto negativo sobre la depresión; este efecto fue mayor para las mujeres que para los hombres. De manera que, se puede suponer que la alta autoestima tiene un efecto protector sobre la depresión. Dicho efecto se observa que es mayor entre las mujeres ESB que entre sus compañeros hombres (Figura 3). 
Efecto de la depresión y la autoestima en la ideación suicida de adolescentes ... / M. LUNA CONTRERAS y C.A. DÁVILA CERVANTES

Tabla 4: Ciudad de México. Coeficientes estandarizados de las variables latentes del Modelo Estructural de la ideación suicida y la autoestima mediado por la depresión en estudiantes de secundaria o bachillerato por sexo y para el total, 2012

\begin{tabular}{|c|c|c|c|}
\hline \multirow[b]{2}{*}{ Variable latente } & \multicolumn{3}{|c|}{$\beta$ estandarizado } \\
\hline & Total & Hombres & Mujeres \\
\hline \multicolumn{4}{|l|}{ Ideación suicida } \\
\hline No podía seguir adelante & $\begin{array}{l}0.770^{* * * *} \\
(0.013)\end{array}$ & $\begin{array}{l}0.778 * * * * \\
(0.018)\end{array}$ & $\begin{array}{l}0.764 * * * * \\
(0.016)\end{array}$ \\
\hline Tenían pensamientos sobre la muerte & $\begin{array}{l}0.830^{* * * * *} \\
(0.011)\end{array}$ & $\begin{array}{l}0.806^{* * * * *} \\
(0.016)\end{array}$ & $\begin{array}{l}0.851 * * * * \\
(0.013)\end{array}$ \\
\hline $\begin{array}{l}\text { Sentían que mi familia estaría mejor si yo } \\
\text { estuviera muerto }\end{array}$ & $\begin{array}{l}0.852^{* * * * *} \\
(0.010)\end{array}$ & $\begin{array}{l}0.853^{* * * *} \\
(0.013)\end{array}$ & $\begin{array}{l}0.849 * * * * \\
(0.013)\end{array}$ \\
\hline Pensé en matarme & $\begin{array}{l}0.814 * * * * \\
(0.011)\end{array}$ & $\begin{array}{l}0.814 * * * * \\
(0.015)\end{array}$ & $\begin{array}{l}0.815^{* * * *} \\
(0.013)\end{array}$ \\
\hline \multicolumn{4}{|l|}{ Autoestima } \\
\hline Siento que tengo buenas cualidades & $\begin{array}{l}0.810^{* * * *} \\
(0.010)\end{array}$ & $\begin{array}{l}0.827 * * * * \\
(0.013)\end{array}$ & $\begin{array}{l}0.793 * * * * \\
(0.012)\end{array}$ \\
\hline $\begin{array}{l}\text { Soy capaz de hacer las cosas tan bien } \\
\text { como casi toda la gente }\end{array}$ & $\begin{array}{l}0.830 * * * * \\
(0.009)\end{array}$ & $\begin{array}{l}0.857 * * * * \\
(0.011)\end{array}$ & $\begin{array}{l}0.803 * * * * \\
(0.011)\end{array}$ \\
\hline $\begin{array}{l}\text { Tengo una actitud positiva hacia mí } \\
\text { mismo/a }\end{array}$ & $\begin{array}{l}0.900 * * * * \\
(0.007)\end{array}$ & $\begin{array}{l}0.910^{* * * *} \\
(0.008)\end{array}$ & $\begin{array}{l}0.889 * * * * \\
(0.010)\end{array}$ \\
\hline $\begin{array}{l}\text { En general estoy satisfecho/a conmigo } \\
\text { mismo/a }\end{array}$ & $\begin{array}{l}0.873 * * * * \\
(0.008)\end{array}$ & $\begin{array}{l}0.877 * * * * \\
(0.010)\end{array}$ & $\begin{array}{l}0.869 * * * * \\
(0.010)\end{array}$ \\
\hline \multicolumn{4}{|l|}{ Depresión } \\
\hline $\begin{array}{l}\text { Sentía que no podía quitarme la tristeza ni } \\
\text { con la ayuda de mi familia o amigos }\end{array}$ & $\begin{array}{l}0.748 * * * * \\
(0.012)\end{array}$ & $\begin{array}{l}0.715^{* * * * *} \\
(0.017)\end{array}$ & $\begin{array}{l}0.766 * * * * \\
(0.013)\end{array}$ \\
\hline Me sentí deprimido/a & $\begin{array}{l}0.795 * * * * \\
(0.010)\end{array}$ & $\begin{array}{l}0.775^{* * * *} \\
(0.014)\end{array}$ & $\begin{array}{l}0.804 * * * * \\
(0.012)\end{array}$ \\
\hline Pensé que mi vida era un fracaso & $\begin{array}{l}0.720 * * * * \\
(0.013)\end{array}$ & $\begin{array}{l}0.707 * * * * \\
(0.018)\end{array}$ & $\begin{array}{c}0.719 \text { **** } \\
(0.014)\end{array}$ \\
\hline Tenía miedo & $\begin{array}{l}0.690 * * * * \\
(0.014)\end{array}$ & $\begin{array}{l}0.681^{* * * * *} \\
(0.019)\end{array}$ & $\begin{array}{c}0.689 * * * * \\
(0.014)\end{array}$ \\
\hline Me sentía solo/a & $\begin{array}{l}0.772 * * * * \\
(0.010)\end{array}$ & $\begin{array}{l}0.744 * * * * \\
(0.015)\end{array}$ & $\begin{array}{c}0.784 * * * * \\
(0.012)\end{array}$ \\
\hline Me sentía triste & $\begin{array}{l}0.818^{* * * * *} \\
(0.009)\end{array}$ & $\begin{array}{l}0.798 * * * * \\
(0.012)\end{array}$ & $\begin{array}{c}0.833 * * * * \\
(0.010)\end{array}$ \\
\hline
\end{tabular}

${ }^{*}$ p-valor $\leq 0.10 ; *$ p-valor $\leq 0.05 ; * * *$-valor $\leq 0.01 ; * * * *$-valor $\leq 0.001$.

Error estándar entre paréntesis.

Fuente: elaboración propia con base en la Encuesta de Consumo de Drogas en Estudiantes Ciudad de México 2012. 
Figura 3: Ciudad de México. Modelo Estructural de la ideación suicida y la autoestima mediado por la depresión en estudiantes de secundaria o bachillerato por sexo y para el total, 2012

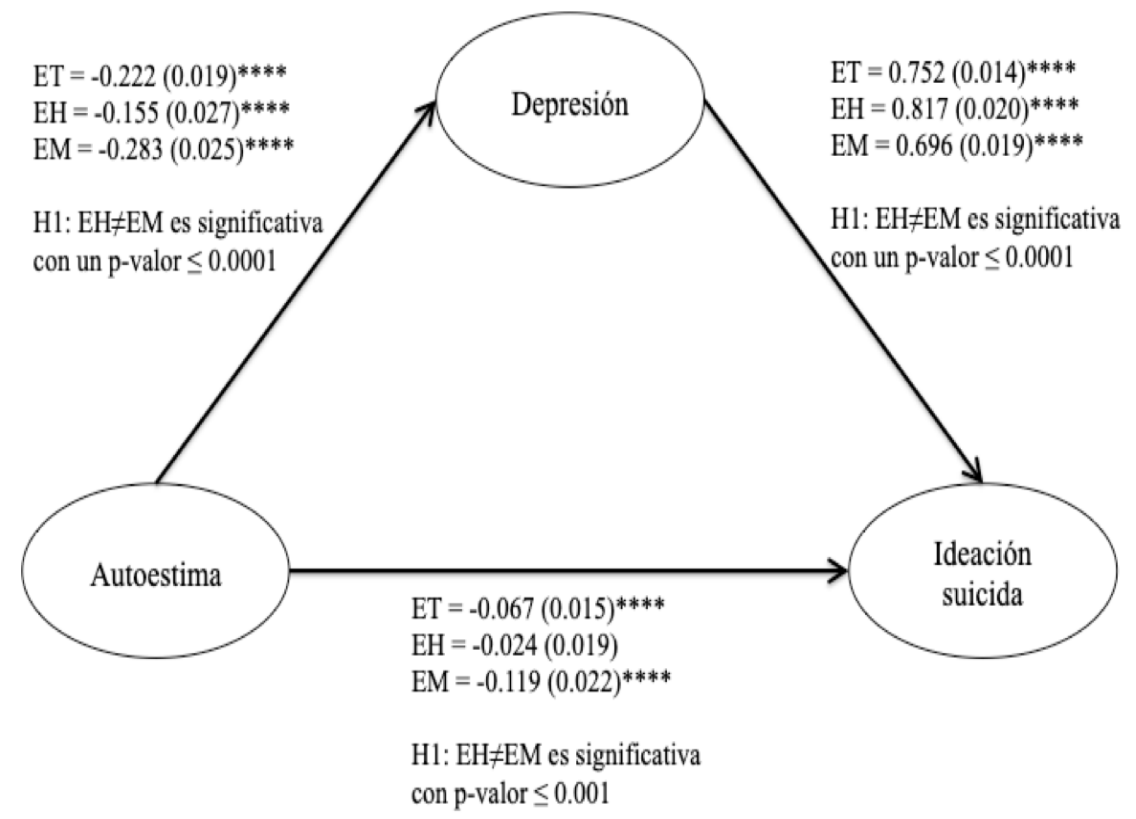

Total: $\mathrm{RMSEA}=0.042 ; \mathrm{CFI}=0.956 ; \mathrm{TLI}=0.946 ; \mathrm{SRMR}=0.039$.

Por sexo: RMSEA $=0.040 ; \mathrm{CFI}=0.952 ; \mathrm{TLI}=0.948 ; \mathrm{SRMR}=0.042$.

ET se refiere al total de estudiantes, EH a los estudiantes hombres y EM a las estudiantes mujeres. Coeficientes estandarizados. Error estándar entre paréntesis.

${ }^{*}$ p-valor $\leq 0.05 ; * *$ p-valor $\leq 0.01 ; * * *$-valor $\leq 0.001 ; * * * *$ p-valor $\leq 0.0001$.

Fuente: elaboración propia con base en la Encuesta de Consumo de Drogas en Estudiantes Ciudad de México 2012.

Por otro lado, el efecto indirecto de la autoestima sobre la ideación suicida mediado por la depresión resultó ser negativo y estadísticamente significativo para el total de los ESB y por sexo. El efecto total de la autoestima sobre la ideación suicida (que corresponde al efecto directo más el efecto indirecto) fue más del doble para las mujeres que para los hombres ESB. De esta manera, se puede observar que los resultados del modelo de SEM muestran que la depresión tuvo un efecto potenciador en la ideación suicida ante niveles más bajos de autoestima. Dicho efecto fue mayor entre los hombres, de tal manera que, para ellos, aun cuando la baja autoestima no tiene un efecto directo sobre la ideación suicida, la autoestima sí tiene un efecto ante la presencia de sintomatología depresiva (Tabla 5). 
Efecto de la depresión y la autoestima en la ideación suicida de adolescentes ... / M. LUNA CONTRERAS y C.A. DÁVILA CERVANTES

Tabla 5: Ciudad de México. Efecto total, directo e indirecto de la autoestima en la ideación suicida en estudiantes de secundaria o bachillerato por sexo y para el total, 2012

\begin{tabular}{|c|c|c|c|c|}
\hline \multirow[b]{2}{*}{ Efectos } & \multicolumn{3}{|c|}{$\beta$ estandarizado } & \multirow{2}{*}{$\begin{array}{c}\text { Diferencia } \\
\text { (Hombres - } \\
\text { Mujeres) } \\
\end{array}$} \\
\hline & Total & Hombres & Mujeres & \\
\hline Total (directo + indirecto) & $\begin{array}{c}-0.234 * * * * \\
(0.020)\end{array}$ & $\begin{array}{c}-0.150 * * * \\
(0.029)\end{array}$ & $\begin{array}{c}-0.316^{* * * * *} \\
(0.027)\end{array}$ & $0.166^{* * * *}$ \\
\hline Directo & $\begin{array}{c}-0.067 * * * * \\
(0.015)\end{array}$ & $\begin{array}{l}-0.024 \\
(0.019)\end{array}$ & $\begin{array}{c}-0.119 * * * * \\
(0.022)\end{array}$ & $0.095 * * *$ \\
\hline $\begin{array}{l}\text { Indirecto } \\
\text { Autoestima en Ideación } \\
\text { suicida (mediada por la } \\
\text { Depresión) }\end{array}$ & $\begin{array}{c}-0.167 * * * * \\
(0.014)\end{array}$ & $\begin{array}{c}-0.127 * * * \\
(0.023)\end{array}$ & $\begin{array}{c}-0.197 * * * * \\
(0.007)\end{array}$ & $0.070^{*}$ \\
\hline
\end{tabular}

\section{Discusión}

La presente investigación tuvo como principal objetivo analizar el efecto de la depresión y la autoestima sobre la ideación suicida en adolescentes ESB de la CDMX en 2012. Entre los principales resultados se observó que las mujeres estudiantes tuvieron una mayor prevalencia que los hombres de síntomas de ideación suicida, y en aquellos relacionados con la depresión y con una baja autoestima; dichas diferencias fueron estadísticamente significativas. Se observó también que, entre los ESB de la CDMX, la autoestima y la depresión tuvieron un efecto directo y estadísticamente significativo en la ideación suicida. Por sexo, se tuvo que la depresión tuvo un mayor efecto sobre la ideación suicida en los hombres; mientras que solamente en las mujeres la autoestima tuvo un efecto directo sobre la ideación suicida. Sin embargo, la autoestima tuvo un efecto negativo y significativo sobre la ideación suicida, tanto en hombres como en mujeres, cuando ésta se encuentra mediada por la depresión.

Se observó que poco más de una tercera parte de los ESB presentaron por lo menos un síntoma de ideación suicida al menos una vez a la semana, con una mayor frecuencia femenina (Wolfe et al., 2019). Un estudio realizado en la Región Metropolitana de Santiago de Chile encontró una prevalencia de ideación suicida en adolescentes casi 60 por ciento (Ventura-Juncá et al., 2010), mientras que en Canadá encontraron una prevalencia de 33 por ciento (Brezo et al., 2007) y en Estados Unidos de 24 por ciento 
(Waldrop et al., 2007). En una revisión sistemática se concluyó que 29.9 por ciento de adolescentes ha tenido ideas suicidas en algún momento de su vida (Evans et al., 2005); mientras que en adolescentes del Área Metropolitana de la CDMX en 2013 se obtuvo una prevalencia de ideación suicida de 15.9 por ciento (Borges et al., 2017). Además, según la OMS de 12 a 25 por ciento de los adolescentes presentan alguna idea frente al suicidio (Ceballos-Ospino et al., 2015). Como se observa, la prevalencia de ideación suicida en adolescentes oscila entre 11.5 y 60 por ciento. Cabe señalar que dichas prevalencias y la aquí presentada no son comparables por la forma en cómo se estimó el constructo latente de ideación suicida. De igual forma, casi dos terceras partes de los adolescentes ESB de la CDM tuvieron al menos un síntoma depresivo; y al igual que la ideación, esta prevalencia fue mayor para las mujeres. Este resultado es similar al encontrado en otro estudio en el cual las mujeres adolescentes presentaron una mayor prevalencia de depresión (Breslau et al., 2017). En cuanto a la autoestima se observó que los hombres tuvieron una mayor autoestima entre los ESB. Esto mismo se ha observado en otras investigaciones y otros contextos (Birndorf et al., 2005; Sánchez-Queija et al., 2017).

La depresión es posiblemente la variable más relacionada con la ideación suicida, mostrando consistentemente una fuerte asociación entre ambas (Primananda y Keliat, 2019). En este estudio se encontró que la presencia de depresión, incrementa la posibilidad que los ESB desarrollen ideación suicida. Esto es consistente con otros estudios previos realizados en adolescentes, quienes tienen un mayor riesgo de desarrollar ideación suicida (Primananda y Keliat, 2019; Hong et al., 2016). Esto se debe a que la depresión se asocia comúnmente con sentimientos de desesperación, impotencia, falta de apoyo social y dificultad para afrontar problemas o dificultades en la vida (Hong et al., 2016). Destaca también, que la depresión tuvo un mayor efecto sobre la ideación suicida para los estudiantes hombres, esto a pesar de que la prevalencia fue mayor en mujeres. Esto resultados abren la puerta para investigaciones futuras sobre el efecto diferencial por sexo que tiene la depresión sobre la ideación suicida. Además, muestran que la depresión tiene un rol central en el desarrollo de comportamiento suicida, por lo que debe abordarse lo antes posible para prevenir la ideación suicida (Primananda y Keliat, 2019).

Por su parte, la autoestima tuvo un efecto significativo sobre la ideación suicida. Esta relación implica que, a mayor autoestima del adolescente, se espera que el riesgo de que desarrolle ideación suicida disminuya. Esto es similar a lo reportado en otras investigaciones sobre el tema (Manani y 
Sharma, 2016; Primananda y Keliat, 2019). Dicha relación entre ambos fenómenos se ha intentado explicar argumentando que los adolescentes con buena autoestima desarrollan un sentido de auto-aceptación y satisfacción con la vida que ayuda a disminuir el deseo de pensar en cometer un suicidio (Primananda y Keliat, 2019). O por el contrario, la ideación suicida puede surgir de una constante percepción negativa de uno mismo, lo que implica que los adolescentes con baja autoestima pueden percibir que vida no vale la pena y a los estresores cotidianos como abrumadores (Manani y Sharma, 2016). La autoestima, por lo tanto, tiene un rol importante en la comprensión de la conducta suicida en los adolescentes (Manani y Sharma, 2016). Sin embargo, es importante aclarar que dicha relación solo se sostuvo para las mujeres ESB de la CDMX.

A pesar de ello, los resultados del modelo de SEM muestran que en ambos sexos la autoestima disminuyó la ideación suicida cuando ésta se encuentra mediada por la depresión. En este sentido, se observó que una autoestima alta tuvo un efecto protector sobre la depresión. Este resultado es consistente con otras investigaciones que han encontrado que una baja autoestima es uno de los principales factores de riesgo para el desarrollo de depresión en adolescentes (Dinger et al., 2017; Nguyen et al., 2019; Rieger et al., 2016). Estos resultados muestran por lo tanto que, en ambos sexos, la baja autoestima predispone al adolescente a la depresión y a su vez, la depresión potencia el riesgo que un adolescente con niveles bajos de autoestima desarrolle ideación suicida, o lo que es lo mismo, una alta autoestima protege al adolescente de la depresión y por lo tanto de la ideación suicida.

El incremento constante de la mortalidad por suicidio en México en los últimos 40 años y el aumento de la conducta suicida en adolescentes representan una llamada de atención sobre la necesidad de implementar acciones de identificación oportuna, una estrategia integral multisectorial de prevención y el estudio detallado de los factores de riesgo asociados con el fenómeno. En este sentido, las investigaciones sobre la ideación suicida son clave para generar evidencia que pueda ser utilizada para la planeación, diseño y evaluación de estrategias, políticas y programas de prevención del suicidio (Ventura-Juncá et al., 2010). Es importante por tanto promover e implementar actividades de prevención en adolescentes y diseñar protocolos de atención, con un enfoque en los principales factores de riesgo de la ideación suicida, que es el inicio del comportamiento suicida que puede culminar en el suicidio consumado. Esto se puede lograr realizando tamizajes las escuelas, con un enfoque en aquellos adolescentes que presentan sintomatología depresiva y autoestima deteriorada. Todo 
ello para identificar a los estudiantes que están en mayor riesgo de presentar ideación suicida y proveerles la atención necesaria oportunamente, a fin de poder romper el ciclo que inicia con la ideación suicida (Pérez-Amezcua et al., 2010; Wolfe et al., 2019).

\section{Limitaciones}

Los resultados del presente estudio si bien son muy relevantes para la toma de acciones enfocadas en la prevención de la ideación suicida, se deben interpretar a la luz de ciertas limitaciones importantes. Primero, el análisis se realizó con base en auto reportes retrospectivos, por lo que es posible que las prevalencias de la depresión, la autoestima y de la ideación suicida estén subestimadas dada su naturaleza estigmatizante. Segundo, se realizó un estudio transversal, con lo cual no se pudo considerar la temporalidad y el orden de ocurrencia de los fenómenos estudiados (por ejemplo, el desarrollo de depresión previo a la ideación suicida) por lo que las asociaciones observadas entre la depresión, la autoestima y la ideación suicida deben ser tomadas con esta limitación en mente. Además, dado el diseño del estudio, no es posible atribuir una asociación causal entre los eventos estudiados. Esto es relevante ya que se ha encontrado que la autoestima (McGee et al., 2001) y la depresión (De Beurs et al., 2019) predicen prospectivamente la ideación suicida, para lo cual sería necesario realizar estudios longitudinales para abordar esta limitación del presente estudio. Una tercera limitación es que los resultados del estudio solamente pueden ser generalizados para los ESB de la CDMX dada la representatividad de la muestra seleccionada en la encuesta utilizada.

\section{CONCLUSIONES}

El suicidio es uno de los principales problemas de salud pública en México, cuya incidencia se ha incrementado desde hace más de 40 años, especialmente en adolescentes y jóvenes. En este sentido, la conducta suicida es un proceso que inicia con la ideación suicida, que tiene una mayor prevalencia en la adolescencia (Rueter et al., 2008) y que puede llegar a incrementar hasta en 12 veces el riesgo de suicidio antes de los 30 años de edad (Reinherz, Tanner, Berger, Beardslee y Fitzmaurice, 2006). En esta investigación se observó que la baja autoestima y la depresión incrementan el riesgo de desarrollo de ideación suicida en los adolescentes ESB de la CDMX. De igual forma, la depresión tuvo un efecto potenciador en la ideación suicida ante niveles bajos de autoestima. Los resultados analizados se espera que aporten evidencia para una mejor comprensión del 
fenómeno de la ideación suicida e identificar áreas de oportunidad para la planeación, diseño y evaluación de estrategias, políticas y programas de prevención del suicidio.

\section{REFERENCIAS BIBLIOGRáFICAS}

Baams, L., Grossman, A. H. y Russell, S. T., 2015, "Minority stress and mechanisms of risk for depression and suicidal ideation among lesbian, gay, and bisexual youth", in Developmental Psychology, 51(5), pp. 688-696. doi: 10.1037/ a0038994

Bilsen, J., 2018, "Suicide and youth: risk factors", in Frontiers in psychiatry, 9(540), pp. 1-5.

Birndorf, S., Ryan, S., Auinger, P. y Aten, M., 2005, "High self-esteem among adolescents: Longitudinal trends, sex differences, and protective factors", in Journal of Adolescent Health, 37(3), pp. 194-201.

Borges, G., Benjet, C., Orozco, R. y Medina-Mora, M.E., 2017, “The growth of suicide ideation, plan and attempt among young adults in the Mexico City metropolitan area", in Epidemiology and Psychiatric Sciences, 26(6), pp. 635-643.

Borges, G., Orozco, R., Benjet, C. y Medina-Mora, M.E., 2010, "Suicidio y conductas suicidas en México: retrospectiva y situación actual", en Salud Pública de México, 52(4), pp. 292-304.

Borges, G., Rosovsky, H., Gómez, C. y Gutiérrez, R., 1996, "Epidemiología del suicidio en México de 1970 a 1994", en Salud Pública de México, 38(3), pp. 197206.

Breslau, J., Gilman, S. E., Stein, B. D., Ruder, T., Gmelin, T. y Miller, E., 2017, "Sex differences in recent first-onset depression in an epidemiological sample of adolescents", en Translational psychiatry, 7(5), pp. e1139-e1139.

Brezo, J., Paris, J. y Barrer, E., 2007, "Natural history of suicidal behaviors in a population-based sample of young adults", in Psychological Medicine, 37(11), pp. $1563-1574$

Ceballos-Ospino, G.A., Suárez-Colorado, Y., Suescún-Arregocés, J., Gamarra-Vega, L.M., González, K.E. y Sotelo-Manjarres, A.P., 2015, "Ideación suicida, depresión y autoestima en adolescentes escolares de Santa Marta", in Duazary, 12(1), pp.15-22.

Cha, C. B., Franz, P. J., M. Guzmán, E., Glenn, C. R., Kleiman, E. M. y Nock, M. K., 2018, “Annual Research Review: Suicide among youth-epidemiology,(potential) etiology, and treatment", in Journal of Child Psychology and psychiatry, 59(4), pp. 460-482.

Chen, I.M., Liao, S.C, Lee, M.B., Wu, C.Y., Lin, P.H., Chen, W.J., 2016, "Risk factors of suicide mortality among multiple attempters: A national registry study in Taiwan", in Journal of the Formosan Medical Association, 115(5), pp. 364-371. 
Crosby, A. E., Han, B., Ortega, L. A. G., Parks, S. E., y Gfroerer, J., 2011, “Suicidal thoughts and behaviors among adults aged 18 years - United States, 2008-2009", in Morbidity and Mortality Weekly Report Surveillance Summaries, 60, pp. 1-22.

De Beurs, D., Ten Have, M., Cuijpers, P. y De Graaf, R., 2019, “The longitudinal association between lifetime mental disorders and first onset or recurrent suicide ideation", in BMC Psychiatry, 19(1), p. 345.

Dinger, U., Ehrenthal, J. C., Nikendei, C. y Schauenburg, H., 2017, "Change in self-esteem predicts depressive symptoms at follow-up after intensive multimodal psychotherapy for major depression", in Clinical psychology \& psychotherapy, 24(5), pp. 1040-1046.

Duarte, T. A., Paulino, S., Almeida, C., Gomes, H. S., Santos, N., \& Gouveia-Pereira, M., 2020, "Self-harm as a predisposition for suicide attempts: A study of adolescents' deliberate self-harm, suicidal ideation, and suicide attempts", in Psychiatry Research, 287(112553), pp. 1-7.

Evans, E., Hawton, K., Rodham, K. y Deeks, J., 2005, “The prevalence of suicidal phenomena in adolescents: a systematic review of population-based studies", in Suicide and Life-Threatening Behavior, 35, pp. 239-50.

González-Forteza, C., Berenzon-Gorn, S., Tello-Granados, A.M., Facio-Flores, D. y Medina-Mora, M.E., 1998, "Ideación suicida y características asociadas en mujeres adolescentes”, en Salud Pública de México, 40(5), pp. 430-437.

González-Forteza, C. y Andrade, P., 1995, "La relación de los hijos con sus progenitores y sus recursos de apoyo: correlación con la sintomatología depresiva y la ideación suicida en los adolescentes mexicanos", en Salud Mental, 18(4), pp. 41-48.

González-Forteza, C., Andrade, P. y Jiménez, A., 1997, "Recursos psicológicos relacionados con el estrés cotidiano en una muestra de adolescentes mexicanos", en Salud Mental, 20(1), pp. 27-35.

González-Forteza, García, G., Medina-Mora, M.A. y Sánchez, M.A., 1998, “Indicadores psicosociales predictores de ideación suicidad en dos generaciones de estudiantes universitarios", en Salud Mental, 21(3), pp. 1-9.

González-Forteza, C., Solís, C., Jiménez, A., Hernández, I., González-González, A., Juárez, F., Medina-Mora, M. E. y Fernández-Valera, H., 2011, “Confiabilidad y validez de la escala de depresión CES-D en un censo de estudiantes de nivel medio superior y superior, en la Ciudad de México", en Salud Mental, 34(1), pp. 53-59.

Harmer, B., Lee, S., \& Saadabadi, A., 2020, "Suicidal Ideation”. StatPearls. Disponible en https://www.ncbi.nlm.nih.gov/books/NBK565877/. Consultado el 18/02/2021.

Hernández-Bringas, H.H. y Flores-Arenales, R., 2011, “El suicidio en México”, en Papeles de Población, 17(68), pp. 69-101.

Hill, R. M., Penner, F., Vanwoerden, S., Mellick, W., Kazimi, I. and Sharp, C., 2019, "Interpersonal trust and suicide ideation among adolescent psychiatric 
inpatients: An indirect effect via perceived burdensomeness", en Suicide and Life-Threatening Behavior, 49(1), pp. 240-252.

Hong, L., Guo, L., Wu, H., Li, P., Xu, Y., Gao, X. y Lu, C., 2016, "Bullying, depression, and suicidal ideation among adolescents in the Fujian Province of China: a cross-sectional study", in Medicine, 95(5), pp. e.2530.

Instituto Nacional de Psiquiatría Ramón de la Fuente Muñiz, 2013, Encuesta de Consumo de Drogas en Estudiantes Ciudad de México 2012. Disponible en http:// www.uade.inpsiquiatria.edu.mx/investigacionesencuestas\%20estudiantes.html (Acceso 14/04/2020)

Kwak, C. W. and Ickovics, J. R., 2019, “Adolescent suicide in South Korea: risk factors and proposed multi-dimensional solution", in Asian journal of psychiatry, 43, pp. 150-153.

Large, M., Corderoy, A. and McHugh, C., 2020, "Is suicidal behaviour a stronger predictor of later suicide than suicidal ideation? A systematic review and meta-analysis", in Australian and New Zealand Journal of Psychiatry, 2020, pp. 1-14.

Lin, C. C., 2019, "Self-Esteem and Depression as Mediators of the Effects of Gratitude on Suicidal Ideation Among Taiwanese College Students", in OMEGA-Journal of Death and Dying, pp.1-15. doi: 10.1177/0030222819892358

López, J. A. y Zavala, M. A., 2019, "Validación del inventario de habilidades políticas de Ferris mediante análisis factorial de segundo orden", en Vargas, D. (coord). Aspectos metodológicos para la investigación social: modelos de ecuaciones estructurales, México, Universidad Nacional Autónoma de Mexico, pp. 85-128.

Manani, P., y Sharma, S., 2016, "Self esteem and suicidal ideation: a correlational study", in MIER Journal of Educational Studies, Trends and Practices, 3(1), pp. 75-83.

Mariño, M. C., Medina-Mora, M. E., Chaparro, J. J. y González-Forteza, C., 1993, "Confiabilidad y Estructura del CES-D en Adolescentes Mexicanos", en Revista Mexicana de Psicología, 10(2), pp. 141-145.

McGee, R., Williams, S. y Nada-Raja S., 2001, "Low self-esteem and hopelessness in childhood and suicidal ideation in early adulthood", in Journal of Abnormal Child Psychology, 29, pp. 281-291.

Nguyen, D. T., Wright, E. P., Dedding, C., Pham, T. T. and Bunders, J., 2019, “Low self-esteem and its association with anxiety, depression, and suicidal ideation in Vietnamese secondary school students: a cross-sectional study", in Frontiers in Psychiatry, 10(698), pp.1-7.

OMS, 2014, Prevención del suicidio un imperativo global. Organización Mundial de la Salud (OMS), Washington D.C., pp. 94.

Orozco, R., Benjet, C., Borges, G., Arce, M. F. M., Ito, D. F., Fleiz, C. and Villatoro, J. A., 2018, "Association between attempted suicide and academic performance indicators among middle and high school students in Mexico: results from a national survey", in Child and adolescent psychiatry and mental health, 12(1), pp.1-10. 
Pérez-Amezcua, B., Rivera-Rivera, L., Atienzo, E.E., De Castro, F., Leyva-López, A. y Chávez-Ayala, R., 2010, "Prevalencia y factores asociados a la ideación e intento suicida en adolescentes de educación media superior de la República mexicana", en Salud Pública de México, 52(4), pp. 324-333.

Primananda, M. y Keliat, B. A., 2019, "Risk and Protective Factors of Suicidal Ideation in Adolescents", in Comprehensive child and adolescent nursing, 42(sup1), pp. 179-188.

Rieger, S., Göllner, R., Trautwein, U. y Roberts, B. W., 2016, “Low self-esteem prospectively predicts depression in the transition to young adulthood: A replication of Orth, Robins, and Roberts (2008)", in Journal of personality and social psychology, 110(1), pp. e16.

Roberts, E., 1980, "Reliability of CES-D Scale in different ethnical contexts", in Psychiatry Research, 2, pp. 125-134.

Rosenberg, M., 1965, Society and the Adolescent Self-image. Princeton, Princeton University Press, 326p.

Rueter, M., Holm, K., McGeorge, C. y Conger, R., 2008, “Adolescent suicidal ideation subgroups and their association with suicidal plans and attempts in young adulthood", in Suicide Life-Threatening Behavior, 38, pp. 564-75.

Sánchez-Queija, I., Oliva, A. y Parra, Á., 2017, "Stability, change, and determinants of self-esteem during adolescence and emerging adulthood", in Journal of Social and Personal Relationships, 34(8), pp. 1277-1294.

Valdez-Santiago, R., Solórzano, E. H., Iñiguez, M. M., Burgos, L. Á., Hernández, H. G., \& González, Á. M., 2018, “Attempted suicide among adolescents in Mexico: prevalence and associated factors at the national level", in Injury prevention, 24(4), pp. 256-261.

Vargas, D., Delice, P. y Donoso, J., 2019, "Encuesta de percepción de violencia en Guerrero 2018: validación y resultados", en Vargas, D. (coord). Aspectos metodológicos para la investigación social: modelos de ecuaciones estructurales, México, Universidad Nacional Autónoma de México, pp. 85-128.

Vargas, H.B. y Saavedra, J.E., 2012, "Factores asociados con la conducta suicida en adolescentes", in Revista de Neuro-Psiquiatría, 75, pp. 19-28.

Ventura-Juncá, R., Carvajal, C., Undurraga, S., Vicuña, P., Egaña, J. y Garib, M. J., 2010, "Prevalencia de ideación e intento suicida en adolescentes de la Región Metropolitana de Santiago de Chile", en Revista médica de Chile, 138(3), pp. 309-315.

Villatoro, J., Quiroz, N., Gutiérrez, M.L., Díaz, M. y Amador, N., 2006, ¿Cómo educamos a nuestros/as hijos/as?, Encuesta de Maltrato Infantil y Factores Asociados. Instituto Nacional de las Mujeres.

Waldrop, A.E., Hanson, R.F., Resnick, H.S., Kilpatrick, D.G., Naugle, A.E. y Saunders, B.E., 2007, "Risk factors for suicidal behavior among a national sample of adolescents: Implications for prevention”, in Journal Trauma Stress, 20(5), pp. 869-79. 
Wolfe, K. L., Nakonezny, P. A., Owen, V. J., Rial, K. V., Moorehead, A. P., Kennard, B. D. y Emslie, G. J., 2019, "Hopelessness as a predictor of suicide ideation in depressed male and female adolescent youth", in Suicide and Life-Threatening Behavior, 49(1), pp. 253-263.

WHO, 2016, Depression: Facts sheets. World Health Organization (WHO). Disponible en http://www.who.int/en/news-room/fact-sheets/detail/depression

Yadegarfard, M., Meinhold-Bergmann, M. E. y Ho, R., 2014, "Family rejection, social isolation, and loneliness as predictors of negative health outcomes (depression, suicidal ideation, and sexual risk behavior) among Thai maletofemale transgender adolescents", in Journal of LGBT Youth, 11, 347-363. doi: 10.1080/19361653.2014.910483

Yildiz, M., Demirhan, E. y Gurbuz, S., 2018, “Contextual socioeconomic disadvantage and adolescent suicide attempts: a multilevel investigation", in Journal of Youth and Adolescence, 48, 802-814. doi: 10.1007/s10964-018-0961-z

\section{RESUMEN CURRICULAR DE LOS AUTORES}

\section{Marisol Luna Contreras}

Doctora en Economía por la Universidad Nacional Autónoma de México (UNAM). Maestra en Población por la Flacso-México y especialista en Estadística Aplicada por el IIMAS. Es profesora e investigadora de la Facultad Latinoamericana de Ciencias Sociales México (FLACSO México). Desde 2010 colabora como consultora en el Observatorio de Mortalidad Materna. Sus temas de interés son: la salud y la mortalidad maternal; y el intento de suicidio en la población de adolescentes y jóvenes. Pertenece al Sistema Nacional de Investigadores.

Dirección electrónica: msluna@flacso.edu.mx.

Registro ORCID: https://orcid.org/0000-0002-7571-3449.

\section{Claudio Alberto Dávila Cervantes}

Doctor en Estudios de Población y maestro en Demografía por El Colegio de México. Su línea de investigación principal es la mortalidad, con enfoque en la mortalidad por causas violentas (homicidios y suicidios), causas crónico-degenerativas (diabetes y enfermedades cardiovasculares) y mortalidad evitable. Es profesor investigador de la Facultad Latinoamericana de Ciencias Sociales México (FLACSO México), co-coordinador del seminario de tesis Población y Salud y miembro del Sistema Nacional de Investigadores (SNI) Nivel I.

Dirección electrónica: claudio.davila@flacso.edu.mx.

Registro ORCID: https://orcid.org/0000-0002-7656-3606.

Artículo recibido el 11 de mayo de 2020 y aprobado el 5 de noviembre de 2020. 